\title{
ERROR ESTIMATES FOR SOME QUASI-INTERPOLATION OPERATORS
}

\author{
RÜDIGER VERFÜRTH ${ }^{1}$
}

\begin{abstract}
We derive explicit bounds on the constants in error estimates for two quasi-interpolation operators which are modifications of the "classical" Clément-operator. These estimates are crucial for making explicit the constants which appear in popular a posteriori error estimates. They are also compared with corresponding estimates for the standard nodal interpolation operator.

Résumé. Pour deux opérateurs d'interpolation de type Clément on donne des bornes explicites sur les constantes dans les estimations d'erreur d'interpolation. Leur valeurs sont importantes pour le calcul des constantes dans les estimations d'erreur a posteriori. Elles sont comparées aux estimations correspondantes pour l'opérateur d'interpolation Lagrangien aux nœuds.
\end{abstract}

AMS Subject Classification. 65N30, 65N15, 65J15.

Received: October 29, 1997.

\section{INTRODUCTION}

In the last decade adaptive finite element methods based on a posteriori error estimators have become an important tool in scientific computing. The error estimators provide easy-to-compute upper bounds on the error of the actual finite element approximation. These upper bounds are based on interpolation error estimates of the following form (cf. Sect. 1.1 and 1.2 in [11]):

$$
\begin{aligned}
\left\|u-I_{h} u\right\|_{L^{2}(T)} & \leq c_{T} h_{T}^{k}\left\|\nabla^{k} u\right\|_{L^{2}\left(\widetilde{\omega}_{T}\right)}, \\
\left\|u-I_{h} u\right\|_{L^{2}(E)} & \leq c_{E} h_{E}^{k-1 / 2}\left\|\nabla^{k} u\right\|_{L^{2}\left(\widetilde{\omega}_{E}\right)}
\end{aligned}
$$

Here, $k \in\{1,2\}, I_{h}$ is some quasi-interpolation operator, $T$ and $E$ are a simplex and a face thereof, $h_{T}$ and $h_{E}$ measure the size of $T$ and $E$, and $\widetilde{\omega}_{T}$ and $\widetilde{\omega}_{E}$ are neighbourhoods of $T$ and $E$ which should be as small as possible. Note that the interpolate $I_{h} u$ never needs to be computed explicitly. Moreover, for problems in two and three space dimensions, one may choose for $I_{h}$ the standard nodal interpolation operator if $k=2$. But the case $k=1$ is the most interesting one, since it does not require any additional elliptic regularity assumptions.

The constants $c_{T}, c_{E}$ appearing in these interpolation error estimates are of great importance for a correct calibration of the a posteriori error estimators. It is the aim of this article to give reasonable explicit estimates of these constants. To this end we analyze two modifications of the quasi-interpolation operator of Clément [7]. The constants $c_{T}$ and $c_{E}$ depend on the element geometry. This dependence is made explicit and computable in terms of a few element characteristics. Our main tools are a trace theorem and suitable Poincaré inequalities.

Keywords and phrases. Interpolation error estimates; quasi-interpolation operators.

1 Fakultät für Mathematik, Ruhr-Universität Bochum, 44780 Bochum, Germany. e-mail: rv@silly.num1.ruhr-uni-bochum.de

(c) EDP Sciences, SMAI 1999 
As a by-product we obtain an apparently new lower bound for the smallest positive eigenvalue of the Laplace operator with Neumann boundary conditions on non-convex domains (cf. Eq. (2.6) and Lem. 4.2 below). For comparison, we also give similar error estimates for the standard nodal interpolation operator applied to $H^{2}$-functions.

The quasi-interpolation operators of Clément [7] and of Scott and Zhang [10] can be analyzed in a similar way by comparing it with the modified Clément-operators. We do not follow this line since the analysis is rather straightforward and since the resulting constants are larger than the corresponding constants for the modified Clément-operators.

For related work we refer to $[3,5,8]$. Angermann [3] computes the constants $c_{T}$ and $c_{E}$ for the quasiinterpolation operator of Scott and Zhang on a uniform triangulation consisting of right-angled isosceles triangles of equal size. The resulting values are larger than the corresponding numbers of Example 2.5 (1) given below. Duran [8] in particular derives estimates for the constant $\widetilde{c}_{P, x}^{(0)}$ of inequality (4.1) below. His technique is different from ours and is based on generalized Taylor expansions. The resulting estimates for $\widetilde{c}_{P, x}^{(0)}$ are larger than ours ( $c f$. the remark at the end of Sect. 4). Carstensen und Funken [5] also consider the quasi-interpolation operator $P_{h}$ of equation (2.11) below. Their interpolation error estimates are also based on Poincaré estimates of the form (4.1). But their technique for establishing these estimates is different from ours. Their approach is based on suitable extension operators whereas our main tool is a reduction to inscribed circular segments (cf. Sect. 4). The resulting interpolation error estimates are rather similar to ours. Carstensen and Funken only treat the two dimensional case but consider general $L^{p}$-spaces. With minor modifications the analysis of Sections $3-6$ below could also be performed within the $L^{p}$-framework. But we choose to stay within the Hilbert space framework since this simplifies the exposition and clarifies the analysis.

The outline of this article is as follows. In Section 2 we introduce the necessary notations and present our main results. In Sections 3 and 4 we prove a trace theorem and give bounds on the constants appearing in some Poincaré inequalities. In Sections 5-7 we prove the results which were announced in Section 2.

\section{Notations And Main Results}

Consider an open, bounded, and connected polyhedron $\Omega$ in $\mathbb{R}^{n}, n \geq 2$, with a Lipschitz boundary $\Gamma$. The boundary $\Gamma$ consists of two disjoint parts $\Gamma_{D}$ and $\Gamma_{N}$ such that $\Gamma=\Gamma_{D} \cup \Gamma_{N} ; \Gamma_{N}$ may be void. Set

$$
H_{D}^{1}(\Omega):=\left\{u \in H^{1}(\Omega): u=0 \text { on } \Gamma_{D}\right\}
$$

where $L^{2}(\Omega)=H^{0}(\Omega)$ and $H^{k}(\Omega), k \in \mathbb{N}$, denote the standard Sobolev spaces (cf. [2]). For any $1 \leq p \leq \infty$ and any subset $\omega$ of $\Omega$ which is measurable with respect to the $n$ - or $(n-1)$-dimensional Lebesgue measure, we denote by $\|.\|_{p ; \omega}$ the standard $L^{p}(\omega)$-norm. The $k$-dimensional Lebesgue measure is denoted by $\mu_{k}$.

Consider an admissible partition $\mathcal{T}_{h}$ of $\Omega$ into $n$-simplices. Here, as usual, admissible means that any two simplices in $\mathcal{T}_{h}$ share at most a complete $k$-face, $0 \leq k \leq n-1$. Moreover, $\mathcal{T}_{h}$ must be consistent with $\Gamma_{N}$, i.e., $\Gamma_{D}$ and $\Gamma_{N}$ each are the union of $(n-1)$-faces of simplices in $\mathcal{T}_{h}$. Denote by $\mathcal{N}_{h}$ and $\mathcal{E}_{h}$ the set of all vertices and $(n-1)$-faces, resp. in $\mathcal{T}_{h}$. Both sets can be decomposed as

$$
\mathcal{N}_{h}=\mathcal{N}_{h, \Omega} \cup \mathcal{N}_{h, N} \cup \mathcal{N}_{h, D}, \quad \mathcal{E}_{h}=\mathcal{E}_{h, \Omega} \cup \mathcal{E}_{h, N} \cup \mathcal{E}_{h, D}
$$

with

$$
\begin{array}{ll}
\mathcal{N}_{h, N}=\left\{x \in \mathcal{N}_{h}: x \in \Gamma_{N}\right\}, & \mathcal{N}_{h, D}=\left\{x \in \mathcal{N}_{h}: x \in \Gamma_{D}\right\} \\
\mathcal{E}_{h, N}=\left\{E \in \mathcal{E}_{h}: E \subset \Gamma_{N}\right\}, & \mathcal{E}_{h, D}=\left\{E \in \mathcal{E}_{h}: E \subset \Gamma_{D}\right\}
\end{array}
$$

For any $S \in \mathcal{T}_{h} \cup \mathcal{E}_{h}$ we denote by $\mathcal{N}(S)$ and $h_{S}$ the set of its vertices and its diameter, respectively. Finally, $\mathcal{E}(T)$ denotes the set of all $(n-1)$-faces of $T \in \mathcal{T}_{h}$. For any vertex $x \in \mathcal{N}_{h}$ denote by $\lambda_{x}$ the nodal basis function 
corresponding to $x$, i.e., the continuous, piecewise linear function w.r.t. $\mathcal{T}_{h}$ which takes the value 1 at $x$ and which vanishes at all other vertices. Set $\omega_{x}:=\operatorname{supp} \lambda_{x} . \omega_{x}$ is the union of all simplices which have $x$ as a vertex. Since $\omega_{x}$ is star-shaped w.r.t. $x$ there is a closed subset $\Sigma_{x}$ of the unit sphere $S^{n-1}$ and a continuous function $r_{x}: \Sigma_{x} \rightarrow \mathbb{R}_{+}^{*}$ such that

$$
\omega_{x}=\left\{x+r \sigma: \sigma \in \Sigma_{x}, 0 \leq r \leq r_{x}(\sigma)\right\}
$$

Set

$$
h_{x}:=\max _{\sigma \in \Sigma_{x}} r_{x}(\sigma), \quad \rho_{x}:=\min _{\sigma \in \Sigma_{x}} r_{x}(\sigma) .
$$

If $x$ is an interior vertex, we have $\Sigma_{x}=S^{n-1}$. Otherwise, $\Sigma_{x}$ is a submanifold of $S^{n-1}$ and its boundary relative to $S^{n-1}$ is a piecewise smooth $(n-2)$-dimensional manifold. For $a \in \mathbb{R}_{+}^{*}$ set

$$
B_{\Sigma_{x}}(a):=\left\{r \sigma: \sigma \in \Sigma_{x}, 0 \leq r \leq a\right\}, B_{\Sigma_{x}}:=B_{\Sigma_{x}}(1) .
$$

If $x$ is an interior vertex, $B_{\Sigma_{x}}$ is the standard unit ball in $\mathbb{R}^{n}$. Otherwise, it is a sector of the unit ball. Note that, for vertices on the boundary, the shape of $B_{\Sigma_{x}}$ only depends on $\Omega$ and not on the particular partition $\mathcal{T}_{h}$. We split $\mathcal{N}_{h, D}$ and $\mathcal{N}_{h, N}$ into disjoint subsets $\mathcal{N}_{h, D}^{(c)}, \mathcal{N}_{h, D}^{(n c)}$ and $\mathcal{N}_{h, N}^{(c)}, \mathcal{N}_{h, N}^{(n c)}$, resp. corresponding to those boundary vertices with a convex $B_{\Sigma_{x}}$ and those with a non-convex $B_{\Sigma_{x}}$. With every vertex $x$ and every simplex $T$ we associate several quantities which describe the local geometry of $\mathcal{T}_{h}$ :

$$
\begin{array}{rlrl}
\kappa_{1, x} & :=\frac{h_{x}}{\rho_{x}}, & \kappa_{2, x} & :=\max _{\substack{T \in T_{h} \\
x \in \mathcal{N}(T)}} \frac{h_{T}}{h_{x}}, \\
\kappa_{3, x} & :=\max _{\substack{T_{1}, T_{2} \in \mathcal{T}_{h} \\
x \in \mathcal{N}\left(T_{1}\right) \cap \mathcal{N}\left(T_{2}\right)}} \frac{\mu_{n}\left(T_{1}\right)}{\mu_{n}\left(T_{2}\right)}, & \kappa_{4, x}:=\max _{\substack{T \in \mathcal{T}_{h}, E \in \mathcal{E}_{h} \\
x \in \mathcal{N}(T) \cap \mathcal{N}(E)}} \frac{\mu_{n-1}(E)}{\mu_{n}(T)} h_{x} . \\
\kappa_{1, T}:=\max _{\substack{x_{1}, x_{2} \in \mathcal{N}(T) \\
x_{1} \neq x_{2}}} \frac{h_{T}}{\left|x_{1}-x_{2}\right|_{2}}, \quad \kappa_{2, T}:=\max _{E \in \mathcal{E}(T)} \frac{\mu_{n-1}(E) h_{T}}{\mu_{n}(T)} .
\end{array}
$$

Here, $|\cdot|_{2}$ denotes the Euclidean norm in $\mathbb{R}^{n}$.

Moreover, we will frequently refer to the following Poincaré constant

$$
c_{P, x}:=\sup \left\{\frac{\|u\|_{2 ; B_{\Sigma_{x}}}}{\|\nabla u\|_{2 ; B_{\Sigma_{x}}}}: u \in H^{1}\left(B_{\Sigma_{x}}\right), \int_{B_{\Sigma_{x}}} u=0\right\} .
$$

Note, that $c_{P, x}^{-2}$ is the smallest positive eigenvalue of the Laplace operator on $B_{\Sigma_{x}}$ with Neumann boundary conditions on $\partial B_{\Sigma_{x}}$. The following estimates for $c_{P, x}$ are given in Lemma 4.2 below:

$$
c_{P, x} \leq\left\{\begin{array}{lll}
5 / 9 & \text { if } x \in \mathcal{N}_{h, \Omega} & \text { and } n=2, \\
2 / \pi & \text { if } x \in \mathcal{N}_{h, \Omega} & \text { and } n \geq 3, \\
2 / \pi & \text { if } x \in \mathcal{N}_{h, N}^{(c)} \cup \mathcal{N}_{h, D}^{(c)} & \text { and } n \geq 2, \\
1.884 & \text { if } x \in \mathcal{N}_{h, N}^{(n c)} \cup \mathcal{N}_{h, D}^{(n c)} & \text { and } n=2, \\
3.602 & \text { if } x \in \mathcal{N}_{h, N}^{(n c)} \cup \mathcal{N}_{h, D}^{(n c)} & \text { and } n=3, \\
\frac{1}{2} 3^{n / 2}\left\{\frac{16}{\pi^{2}}+\frac{2}{n} \max \left\{\frac{2}{n+2}, \frac{1}{n-2}\right\}\right\}^{1 / 2} & \text { if } x \in \mathcal{N}_{h, D}^{(n c)} \cup \mathcal{N}_{h, N}^{(n c)} & \text { and } n \geq 4 .
\end{array}\right.
$$


In order to formulate our main results in a compact form we introduce two functions $K_{1, n}, K_{2, n} \in C\left(\mathbb{R}_{+}^{*}, \mathbb{R}\right)$ and a number $c_{x}$ by

$$
\begin{aligned}
K_{1, n}(z) & :=4 z^{n-2}-3 z^{-2}, \\
K_{2, n}(z) & := \begin{cases}\max \left\{\frac{1}{2}-\frac{1}{2} z^{-2}, \ln z-\frac{1}{2}+\frac{1}{2} z^{-2}\right\}, & \text { if } n=2 \\
\max \left\{\frac{4}{n(n+2)}\left[z^{n-2}-z^{-2}\right], \frac{2}{n-2}\left[\frac{1}{n} z^{n-2}-\frac{1}{2}+\frac{n-2}{2 n} z^{-2}\right]\right\}, & \text { if } n \geq 3,\end{cases} \\
c_{x} & := \begin{cases}2 / \pi, & \text { if } \omega_{x} \text { is convex, } \\
\left\{c_{P, x}^{2} K_{1, n}\left(\kappa_{1, x}\right)+K_{2, n}\left(\kappa_{1, x}\right)\right\}^{1 / 2}, & \text { otherwise. }\end{cases}
\end{aligned}
$$

Denote by $\mathbb{P}_{k}, k \in \mathbb{N}$, the set of all polynomials of degree at most $k$ and set

$$
\begin{aligned}
S_{h} & :=\left\{v \in C(\bar{\Omega}):\left.v\right|_{T} \in \mathbb{P}_{1} \quad \forall T \in \mathcal{T}_{h}\right\} \subset H^{1}(\Omega), \\
S_{h, D} & :=\left\{v \in S_{h}: v=0 \text { on } \Gamma_{D}\right\} \subset H_{D}^{1}(\Omega) .
\end{aligned}
$$

For $k \in\{0,1\}$ and $x \in \mathcal{N}_{h}$ we define $\pi_{x}^{(k)}: L^{2}\left(\omega_{x}\right) \rightarrow \mathbb{P}_{k}$ by

$$
\pi_{x}^{(0)} u:=\frac{1}{\mu_{n}\left(\omega_{x}\right)} \int_{\omega_{x}} u
$$

and

$$
\pi_{x}^{(1)} u:=a_{0}+\sum_{i=1}^{n} a_{i} x_{i}
$$

with

$$
\begin{aligned}
a_{i} & :=\frac{1}{\mu_{n}\left(\omega_{x}\right)} \int_{\omega_{x}} \frac{\partial u}{\partial x_{i}} \quad, 1 \leq i \leq n, \\
a_{0} & :=\frac{1}{\mu_{n}\left(\omega_{x}\right)} \int_{\omega_{x}}\left\{u-\sum_{i=1}^{n} a_{i} x_{i}\right\} .
\end{aligned}
$$

With the help of $\pi_{x}^{(0)}$ and $\pi_{x}^{(1)}$ we can define two quasi-interpolation operators $P_{h}$ and $Q_{h}$ of $H_{D}^{1}(\Omega)$ into $S_{h, D}$ by

$$
P_{h} u:=\sum_{x \in \mathcal{N}_{h, \Omega} \cup \mathcal{N}_{h, N}} \lambda_{x} \pi_{x}^{(0)} u
$$

and

$$
Q_{h} u:=\sum_{x \in \mathcal{N}_{h, \Omega} \cup \mathcal{N}_{h, N}} \lambda_{x} \pi_{x}^{(1)} u(x) .
$$

These operators are modifications of the quasi-interpolation operator of Clément (cf. [7]). The standard nodal interpolation operator is given by

$$
\Pi_{h} u:=\sum_{x \in \mathcal{N}_{h, \Omega} \cup \mathcal{N}_{h, N}} \lambda_{x} u(x)
$$


Proposition 2.1. For all $u \in H_{D}^{1}(\Omega)$, all $T \in \mathcal{T}_{h}$, and all $E \in \mathcal{E}_{h}$ we have

$$
\begin{aligned}
\left\|u-P_{h} u\right\|_{2 ; T} & \leq \sum_{x \in \mathcal{N}(T)} c_{T ; x}^{(P)} h_{x}\|\nabla u\|_{2 ; \omega_{x}} \\
\left\|u-P_{h} u\right\|_{2 ; E} & \leq \sum_{x \in \mathcal{N}(E)} c_{E ; x}^{(P)} h_{x}^{1 / 2}\|\nabla u\|_{2 ; \omega_{x}}
\end{aligned}
$$

with

$$
\begin{aligned}
c_{T ; x}^{(P)} & = \begin{cases}c_{x}, & \text { if } x \in \mathcal{N}_{h, \Omega} \cup \mathcal{N}_{h, N} \\
c_{x}+\left\{\frac{1}{n+2} \kappa_{3, x}\right\}^{1 / 2}\left\{c_{x}+1\right\}, & \text { if } x \in \mathcal{N}_{h, D}\end{cases} \\
c_{E ; x}^{(P)} & = \begin{cases}\left\{\frac{1}{n} \kappa_{4, x}\right\}^{1 / 2}\left\{c_{x}+1\right\}, & \text { if } x \in \mathcal{N}_{h, \Omega} \cup \mathcal{N}_{h, N}, \\
2\left\{\frac{1}{n} \kappa_{4, x}\right\}^{1 / 2}\left\{c_{x}+1\right\}, & \text { if } x \in \mathcal{N}_{h, D} .\end{cases}
\end{aligned}
$$

Proposition 2.2. For all $u \in H^{2}(\Omega) \cap H_{D}^{1}(\Omega)$, all $T \in \mathcal{T}_{h}$, and all $E \in \mathcal{E}_{h}$ we have

$$
\begin{aligned}
\left\|u-Q_{h} u\right\|_{2 ; T} & \leq \sum_{x \in \mathcal{N}(T)} c_{T ; x}^{(Q)} h_{x}^{2}\left\|\nabla^{2} u\right\|_{2 ; \omega_{x}} \\
\left\|u-Q_{h} u\right\|_{2 ; E} & \leq \sum_{x \in \mathcal{N}(E)} c_{E ; x}^{(Q)} h_{x}^{3 / 2}\left\|\nabla^{2} u\right\|_{2 ; \omega_{x}}
\end{aligned}
$$

with

$$
\begin{aligned}
& c_{T ; x}^{(Q)}= \begin{cases}\left\{\sqrt{2}+\frac{1}{n+1}\right\} c_{x}^{2} & \text { if } x \in \mathcal{N}_{h, \Omega} \cup \mathcal{N}_{h, N}, \\
\left\{\sqrt{2}+\frac{1}{n+1}\right\} c_{x}^{2}+n\left\{\frac{2}{n+2} \kappa_{3, x}\right\}^{1 / 2}\left\{c_{x}^{2}+\kappa_{2, x} c_{x}\right\} & \text { if } x \in \mathcal{N}_{h, D},\end{cases} \\
& c_{E ; x}^{(Q)}= \begin{cases}\left\{\sqrt{2}+\frac{1}{n}\right\}\left\{n \kappa_{4, x}\right\}^{1 / 2}\left\{c_{x}^{2}+\kappa_{2, x} c_{x}\right\} & \text { if } x \in \mathcal{N}_{h, \Omega} \cup \mathcal{N}_{h, N}, \\
\left\{2 \sqrt{2}+\frac{1}{n}\right\}\left\{n \kappa_{4, x}\right\}^{1 / 2}\left\{c_{x}^{2}+\kappa_{2, x} c_{x}\right\} & \text { if } x \in \mathcal{N}_{h, D} .\end{cases}
\end{aligned}
$$

Remark 2.3. Estimates similar to those of Proposition 2.1 can be proven for $Q_{h}$, too. The constants, however, are greater than $c_{T ; x}^{(P)}$ and $c_{E ; x}^{(P)}$.

Proposition 2.4. Assume that $n \in\{2,3\}$. For all $u \in H^{2}(\Omega) \cap H_{D}^{1}(\Omega)$, all $T \in \mathcal{T}_{h}$, and all $E \in \mathcal{E}(T)$ we have

$$
\begin{gathered}
\left\|u-\Pi_{h} u\right\|_{2 ; T} \leq c_{T}^{(I)} h_{T}^{2}\left\|\nabla^{2} u\right\|_{2 ; T} \\
\left\|u-\Pi_{h} u\right\|_{2 ; E} \leq c_{E}^{(I)} h_{T}^{3 / 2}\left\|\nabla^{2} u\right\|_{2 ; T}
\end{gathered}
$$

with

$$
\begin{aligned}
& c_{T}^{(I)}=\frac{2(n+1)}{4-n}, \\
& c_{E}^{(I)}=\frac{2(n+1)}{4-n}\left\{n \kappa_{2, T}\right\}^{1 / 2}\left\{1+\kappa_{1, T}\right\} .
\end{aligned}
$$


TABLE 1.

\begin{tabular}{|c|c|c|c|c|c|}
\hline & $\mathcal{N}_{h, \Omega}$ & $\mathcal{N}_{h, N}^{(c)}$ & $\mathcal{N}_{h, D}^{(c)}$ & $\mathcal{N}_{h, N}^{(n c)}$ & $\mathcal{N}_{h, D}^{(n c)}$ \\
\hline$c_{T ; x}^{(P)}$ & 0.637 & 0.637 & 1.456 & 3.452 & 5.678 \\
$c_{E ; x}^{(P)}$ & 2.316 & 2.316 & 4.631 & 6.297 & 12.593 \\
\hline$c_{T ; x}^{(Q)}$ & 0.710 & 0.710 & 2.558 & 20.825 & 44.582 \\
$c_{E ; x}^{(Q)}$ & 7.075 & 7.075 & 12.301 & 90.949 & 158.142 \\
\hline & & & & & \\
\hline$c_{T}^{(I)}$ & 3 & 3 & 3 & 3 & 3 \\
$c_{E}^{(I)}$ & 20.486 & 20.486 & 20.486 & 20.486 & 20.486 \\
\hline
\end{tabular}

Example 2.5. (1) Consider a uniform triangulation, i.e. $n=2$, consisting of isosceles, right-angled triangles with short sides of length $h$. Then all triangles have equal area $\frac{1}{2} h^{2}$ and

$$
h_{x} \in\{h, \sqrt{2} h\}, \quad \rho_{x} \in\left\{\frac{1}{\sqrt{2}} h, h\right\} .
$$

This yields the estimates

$$
\begin{array}{ll}
\kappa_{1, x} \leq 2, & \kappa_{2, x} \leq \sqrt{2}, \\
\kappa_{3, x}=1, & \kappa_{4, x} \leq 4, \\
K_{1,2}\left(\kappa_{1, x}\right) \leq \frac{13}{4}, & K_{2,2}\left(\kappa_{1, x}\right) \leq \frac{3}{8} \\
\kappa_{1, T}=\sqrt{2}, & \kappa_{2, T}=4,
\end{array}
$$

and

$$
c_{x} \leq \begin{cases}0.637, & \text { if } x \in \mathcal{N}_{h, \Omega} \cup \mathcal{N}_{h, D}^{(c)} \cup \mathcal{N}_{h, N}^{(c)}, \\ 3.452, & \text { if } x \in \mathcal{N}_{h, D}^{(n c)} \cup \mathcal{N}_{h, N}^{(n c)}\end{cases}
$$

Consequently, we obtain the following values for the constants of Propositions 2.1, 2.2, 2.4 (see Tab. 1).

(2) Consider a locally refined triangulation, i.e. $n=2$, consisting of isosceles, right-angled triangles. (At first sight this condition seems to be very restrictive. When using appropriate refinement rules (cf. Sect. 4.1 in [11]) it, however, allows strongly refined meshes with very sharp refinement zones.) We first observe that, due to the definitions of $h_{x}$ and $\rho_{x}$, the shortest edge of any triangle $T$, which has $x$ as a vertex, is not smaller than $\rho_{x}$, and not larger than $h_{x}$. Hence, we have for all such triangles

$$
h_{T} \leq \sqrt{2} h_{x}, \quad \frac{1}{2} \rho_{x}^{2} \leq \mu_{2}(T) \leq \frac{1}{2} h_{x}^{2} .
$$


This implies that

$$
\kappa_{2, x} \leq \sqrt{2}, \quad \kappa_{3, x} \leq \kappa_{1, x}^{2}, \quad \kappa_{4, x} \leq 2 \kappa_{1, x}^{2} .
$$

Next, we look for a configuration which yields a $\kappa_{1, x}$ which is as large as possible. Elementary geometrical considerations show that such a configuration is obtained as follows: Let $T_{1}$ be a triangle such that $x$ is on its longest edge and such that this edge has length $h_{x}$. Having constructed $T_{i}, i \geq 1$, we proceed in the counterclockwise sense to $T_{i+1}$ by gluing it to the shortest edge of $T_{i}$ which emanates from $x$ such that this edge becomes the longest edge of $T_{i+1}$. Each step of this procedure reduces the length of the longest edge by a factor $\sqrt{2}$. If $x$ is a non-convex boundary node and if we admit slit domains, we thus obtain the maximal value $\kappa_{1, x}=16$. If $x$ is a convex boundary node, we thus get the maximal value $\kappa_{1, x}=4$. Finally, if $x$ is an interior node, the triangles $T_{1}$ and $T_{8}$ need to match. We therefore obtain in this case the maximal value $\kappa_{1, x}=4$. Summarizing, the maximal values for interior and convex boundary nodes are

$$
\begin{array}{ll}
\kappa_{1, x} \leq 4, & \kappa_{2, x} \leq \sqrt{2} \\
\kappa_{3, x} \leq 16, & \kappa_{4, x} \leq 32, \\
K_{1,2}\left(\kappa_{1, x}\right) \leq \frac{61}{16}, & K_{2,2}\left(\kappa_{1, x}\right) \leq 0.918
\end{array}
$$

for non-convex boundary nodes they are

$$
\begin{array}{ll}
\kappa_{1, x} \leq 16, & \kappa_{2, x} \leq \sqrt{2}, \\
\kappa_{3, x} \leq 256, & \kappa_{4, x} \leq 512, \\
K_{1,2}\left(\kappa_{1, x}\right) \leq \frac{1021}{256}, & K_{2,2}\left(\kappa_{1, x}\right) \leq 2.275 .
\end{array}
$$

Hence, we have

$$
c_{x} \leq \begin{cases}0.637, & \text { if } x \in \mathcal{N}_{h, \Omega} \cup \mathcal{N}_{h, D}^{(c)} \cup \mathcal{N}_{h, N}^{(c)}, \\ 4.054, & \text { if } x \in \mathcal{N}_{h, N}^{(n c)} \cup \mathcal{N}_{h, D}^{(n c)} .\end{cases}
$$

The quantities $\kappa_{1, T}$ and $\kappa_{2, T}$, on the other hand, are the same as in the first part. Consequently, we obtain the following values for the constants of Propositions 2.1, 2.2, and 2.4 (see Tab. 2).

(3) Note that in both tables, the numbers for non-convex boundary vertices correspond to the worst case of a vanishing exterior angle, i.e., of a slit domain. If a positive lower bound for the exterior angle is given, the value of $c_{P, x}$ in (2.6) and, in the case of a non-uniform triangulation, the numbers $\kappa_{1, x}, \ldots, \kappa_{4, x}$ diminish. The quantities $c_{T ; x}^{(P)}, \ldots$ are then reduced correspondingly. Both tables show that elements sharing a non-convex boundary vertex should preferably be of equal size and shape.

\section{A TRACE THEOREM}

Denote by $e_{i}, 1 \leq i \leq n$, the $i$ th unit vector in $\mathbb{R}^{n}$ and by $\widehat{T}$ the reference $n$-simplex with vertices $e_{1}, \ldots, e_{n}$ and $e_{n+1}:=0$. Set

$$
\begin{aligned}
\widehat{E}_{i} & :=\left\{x \in \widehat{T}: x_{i}=0\right\}, \quad 1 \leq i \leq n, \\
\widehat{E}_{n+1} & :=\left\{x \in \widehat{T}:|x|_{1}=1\right\},
\end{aligned}
$$

where $|\cdot|_{1}$ denotes the standard $l^{1}$-norm in $\mathbb{R}^{n}$. 
TABle 2.

\begin{tabular}{|c|c|c|c|c|c|}
\hline & $\mathcal{N}_{h, \Omega}$ & $\mathcal{N}_{h, N}^{(c)}$ & $\mathcal{N}_{h, D}^{(c)}$ & $\mathcal{N}_{h, N}^{(n c)}$ & $\mathcal{N}_{h, D}^{(n c)}$ \\
\hline$c_{T ; x}^{(P)}$ & 0.637 & 0.637 & 3.911 & 4.054 & 44.486 \\
$c_{E ; x}^{(P)}$ & 6.548 & 6.548 & 13.096 & 80.865 & 161.728 \\
\hline$c_{T ; x}^{(Q)}$ & 0.710 & 0.710 & 8.102 & 28.721 & 530.329 \\
$c_{E ; x}^{(Q)}$ & 20.010 & 20.010 & 34.792 & 1357.906 & 2361.121 \\
\hline & & & & & \\
\hline$c_{T}^{(I)}$ & 3 & 3 & 3 & 3 & 3 \\
$c_{E}^{(I)}$ & 20.486 & 20.486 & 20.486 & 20.486 & 20.486 \\
\hline
\end{tabular}

Lemma 3.1. For any $v \in H^{1}(\widehat{T})$ which vanishes on $\widehat{E}_{n+1}$ and any $i \in\{1, \ldots, n\}$ we have

$$
\|v\|_{2 ; \widehat{E}_{i}} \leq\left\|\frac{\partial}{\partial x_{i}} v\right\|_{2 ; \widehat{T}}
$$

Proof. Fix an $i \in\{1, \ldots, n\}$ and a $v \in H^{1}(\widehat{T})$ which vanishes on $\widehat{E}_{n+1}$. Then we have for all $x^{\prime} \in \widehat{E}_{i}$

$$
\begin{aligned}
\left|v\left(x^{\prime}\right)\right|^{2} & =\left|v\left(x^{\prime}\right)-v\left(x^{\prime}+\left[1-\left|x^{\prime}\right|_{1}\right] e_{i}\right)\right|^{2} \\
& =\left|\int_{0}^{1-\left|x^{\prime}\right|_{1}} \frac{\partial}{\partial x_{i}} v\left(x^{\prime}+t e_{i}\right) \mathrm{dt}\right|^{2} \\
& \leq \int_{0}^{1-\left|x^{\prime}\right|_{1}}\left|\frac{\partial}{\partial x_{i}} v\left(x^{\prime}+t e_{i}\right)\right|^{2} \mathrm{dt} .
\end{aligned}
$$

Integrating over $\widehat{E}_{i}$ and invoking Fubini's theorem, this proves the assertion.

Lemma 3.2. For any $T \in \mathcal{T}_{h}$, any $E \in \mathcal{E}(T)$, any $x \in \mathcal{N}(E)$, and any $v \in H^{1}(T)$ we have

$$
\left\|\lambda_{x} v\right\|_{2 ; E} \leq\left\{\frac{\mu_{n-1}(E)}{n \mu_{n}(T)}\right\}^{1 / 2}\left\{\|v\|_{2 ; T}+h_{x}\|\nabla v\|_{2 ; T}\right\}
$$

and

$$
\|v\|_{2 ; E} \leq\left\{n \frac{\mu_{n-1}(E)}{\mu_{n}(T)}\right\}^{1 / 2}\left\{\|v\|_{2 ; T}+h_{T}\|\nabla v\|_{2 ; T}\right\}
$$

Proof. Denote by $F: \widehat{T} \rightarrow T$ an affine mapping which maps $\widehat{T}$ onto $T$ and $e_{n+1}$ onto $x$. Then $E$ is the image of some $\widehat{E}_{i}, 1 \leq i \leq n$, and $\lambda_{x} \circ F=\widehat{\lambda}_{n+1}$ where $\widehat{\lambda}_{n+1}$ is the $(n+1)$-st barycentric coordinate of $\widehat{T}$. Set $\widehat{v}:=v \circ F$. 
Since $\widehat{\lambda}_{n+1} \widehat{v}$ vanishes on $\widehat{E}_{n+1}$ we may apply Lemma 3.1 and obtain

$$
\begin{aligned}
\left\|\lambda_{x} v\right\|_{2 ; E} & =\left\{\frac{\mu_{n-1}(E)}{\mu_{n-1}\left(\widehat{E}_{i}\right)}\right\}^{1 / 2}\left\|\widehat{\lambda}_{n+1} \widehat{v}\right\|_{2 ; \widehat{E}_{i}} \\
& \leq\left\{\frac{\mu_{n-1}(E)}{\mu_{n-1}\left(\widehat{E}_{i}\right)}\right\}^{1 / 2}\left\|\frac{\partial}{\partial x_{i}}\left(\widehat{\lambda}_{n+1} \widehat{v}\right)\right\|_{2 ; \widehat{T}} \\
& \leq\left\{\frac{\mu_{n-1}(E)}{\mu_{n-1}\left(\widehat{E}_{i}\right)}\right\}^{1 / 2}\left\{\left\|\widehat{v} \frac{\partial}{\partial x_{i}} \widehat{\lambda}_{n+1}\right\|_{2 ; \widehat{T}}+\left\|\widehat{\lambda}_{n+1} \frac{\partial}{\partial x_{i}} \widehat{v}\right\|_{2 ; \widehat{T}}\right\} .
\end{aligned}
$$

Since $\frac{\partial}{\partial x_{i}} \widehat{\lambda}_{n+1}=-e_{i}$ and $\left\|\widehat{\lambda}_{n+1}\right\|_{\infty ; \widehat{T}}=1$ this yields

$$
\left\|\lambda_{x} v\right\|_{2 ; E} \leq\left\{\frac{\mu_{n-1}(E)}{\mu_{n-1}\left(\widehat{E}_{i}\right)}\right\}^{1 / 2}\left\{\|\widehat{v}\|_{2 ; \widehat{T}}+\left\|\frac{\partial}{\partial x_{i}} \widehat{v}\right\|_{2 ; \widehat{T}}\right\}
$$

Transforming back to $\widehat{T}$ we get

$$
\|\widehat{v}\|_{2 ; \widehat{T}}=\left\{\frac{\mu_{n}(\widehat{T})}{\mu_{n}(T)}\right\}^{1 / 2}\|v\|_{2 ; T}
$$

and

$$
\begin{aligned}
\left\|\frac{\partial}{\partial x_{i}} \widehat{v}\right\|_{2 ; \widehat{T}} & =\left\{\frac{\mu_{n}(\widehat{T})}{\mu_{n}(T)}\right\}^{1 / 2}\left\|\nabla v \cdot D F \cdot e_{i}\right\|_{2 ; T} \\
& \leq\left\{\frac{\mu_{n}(\widehat{T})}{\mu_{n}(T)}\right\}^{1 / 2}|y-x|_{2}\|\nabla v\|_{2 ; T} \\
& \leq\left\{\frac{\mu_{n}(\widehat{T})}{\mu_{n}(T)}\right\}^{1 / 2} h_{x}\|\nabla v\|_{2 ; T} .
\end{aligned}
$$

Here, $y$ denotes the vertex of $T$, which is not a vertex of $E$, and $|\cdot|_{2}$ is the Euclidean norm in $\mathbb{R}^{n}$. Since $\mu_{n}(\widehat{T})=\frac{1}{n !}$ and $\mu_{n-1}\left(\widehat{E}_{i}\right)=\frac{1}{(n-1) !}$ this proves the first estimate of the lemma.

Since in the first estimate of the lemma $h_{x}$ may be replaced by $h_{T}$, the second estimate of the lemma follows from the first one, the triangle inequality, and the identity

$$
v=\sum_{y \in \mathcal{N}(E)} \lambda_{y} v \text { on } E
$$

\section{Some Poincaré inequalities}

Consider an arbitrary vertex $x \in \mathcal{N}_{h}$ and a $k \in\{0,1\}$. We want to derive explicit bounds on the quantity $\widetilde{c}_{P, x}^{(k)}$ in the Poincaré inequality

$$
\left\|u-\pi_{x}^{(k)} u\right\|_{2 ; \omega_{x}} \leq \widetilde{c}_{P, x}^{(k)}\left\|\nabla^{k+1} u\right\|_{2 ; \omega_{x}} \quad \forall u \in H^{k+1}\left(\omega_{x}\right) .
$$

Using the results of [9] this is an easy task when $\omega_{x}$ is convex. For general $\omega_{x}$, however, the situation is much more complex. A major difficulty lies in the fact that $\omega_{x}$ varies with the vertex $x$ and that there is no fixed reference configuration. One could overcome this difficulty by claiming that there is a small number of reference configurations such that every $\omega_{x}$ is affine equivalent to one of them. Then one could estimate $\widetilde{c}_{P, x}^{(k)}$ for the reference configurations. This approach seems not satisfactory to us. Another possibility would be to imbed every $\omega_{x}$ into a simple larger domain, e.g., a ball, and to construct suitable extension operators from $\omega_{x}$ 
to the larger domain. Then one could estimate $\widetilde{c}_{P, x}^{(k)}$ for the larger domain. This is the approach of [5]. We instead follow another strategy: in Lemma 4.1 we give an explicit estimate of the $L^{2}\left(\omega_{x}\right)$-norm by the $H^{1}\left(\omega_{x}\right)$ semi-norm and the $L^{2}\left(x+B_{\Sigma_{x}}\left(\rho_{x}\right)\right)$-norm. Thus, we only have to estimate $\widetilde{c}_{P, x}^{(k)}$ for balls and segments thereof. This is a relatively easy task and is accomplished in Lemma 4.2.

Lemma 4.1. For all $x \in \mathcal{N}_{h}$ and all $u \in H^{1}\left(\omega_{x}\right)$ we have

$$
\|u\|_{2 ; \omega_{x}}^{2} \leq K_{1, n}\left(\kappa_{1, x}\right) \kappa_{1, x}^{2}\|u\|_{2 ; x+B_{\Sigma_{x}}\left(\rho_{x}\right)}^{2}+K_{2, n}\left(\kappa_{1, x}\right) h_{x}^{2}\|\nabla u\|_{2 ; \omega_{x}}^{2},
$$

where the functions $\kappa_{1, n}$ and $\kappa_{2, n}$ are defined in equation (2.7).

Proof. Since the Lebesgue integral is translation invariant, we may assume that the vertex $x$ is the origin. Let $0<\rho<\rho_{x}$ be arbitrary and set, for abbreviation, $B_{\rho}:=B_{\Sigma_{x}}(\rho)$. Obviously, we have

$$
\|u\|_{2 ; \omega_{x}}^{2}=\|u\|_{2 ; \omega_{x} \backslash B_{\rho}}^{2}+\|u\|_{2 ; B_{\rho}}^{2} .
$$

From equation (2.1) we conclude that

$$
\begin{aligned}
\|u\|_{2 ; \omega_{x} \backslash B_{\rho}}^{2} & =\int_{\Sigma_{x}} \int_{\rho}^{r_{x}(\sigma)} s^{n-1}|u(s \sigma)|^{2} \mathrm{~d} s \mathrm{~d} S(\sigma) \\
& =\int_{\Sigma_{x}} \int_{\rho}^{r_{x}(\sigma)} s^{n-1}|u(s \sigma)-u(\rho \sigma)+u(\rho \sigma)|^{2} \mathrm{~d} s \mathrm{~d} S(\sigma) \\
& \leq 2 \int_{\Sigma_{x}} \int_{\rho}^{r_{x}(\sigma)} s^{n-1}|u(s \sigma)-u(\rho \sigma)|^{2} \mathrm{~d} s \mathrm{~d} S(\sigma)+2 \int_{\Sigma_{x}} \int_{\rho}^{r_{x}(\sigma)} s^{n-1}|u(\rho \sigma)|^{2} \mathrm{~d} s \mathrm{~d} S(\sigma) \\
& =: S_{1}+S_{2} .
\end{aligned}
$$

Here, $\mathrm{d} S$ denotes the $(n-1)$-dimensional surface element on $S^{n-1}$. From definition $(2.2)$ of $h_{x}$ we get

$$
\begin{aligned}
S_{2} & =2 \int_{\Sigma_{x}} \int_{\rho}^{r_{x}(\sigma)} s^{n-1}|u(\rho \sigma)|^{2} \mathrm{~d} s \mathrm{~d} S(\sigma) \\
& =2 \int_{\Sigma_{x}}\left\{\rho^{n-1}|u(\rho \sigma)|^{2} \int_{\rho}^{r_{x}(\sigma)}\left(\frac{s}{\rho}\right)^{n-1} \mathrm{~d} s\right\} \mathrm{d} S(\sigma) \\
& =2 \int_{\Sigma_{x}} \rho^{n-1}|u(\rho \sigma)|^{2} \frac{\rho}{n}\left[\left(\frac{r_{x}(\sigma)}{\rho}\right)^{n}-1\right] \mathrm{d} S(\sigma) \\
& \leq \frac{2 \rho}{n}\left[\left(\frac{h_{x}}{\rho}\right)^{n}-1\right]\|u\|_{2 ; \partial B_{\rho}}^{2} .
\end{aligned}
$$

On the other hand, we conclude from Cauchy-Schwarz' inequality that

$$
\begin{aligned}
S_{1} & =2 \int_{\Sigma_{x}} \int_{\rho}^{r_{x}(\sigma)} s^{n-1}|u(s \sigma)-u(\rho \sigma)|^{2} \mathrm{~d} s \mathrm{~d} S(\sigma) \\
& =2 \int_{\Sigma_{x}} \int_{\rho}^{r_{x}(\sigma)} s^{n-1}\left|\int_{\rho}^{s} \frac{\partial}{\partial r} u(r \sigma) d r\right|^{2} \mathrm{~d} s \mathrm{~d} S(\sigma) \\
& \leq 2 \int_{\Sigma_{x}} \int_{\rho}^{r_{x}(\sigma)} s^{n-1}\left\{\int_{\rho}^{s} r^{n-1}\left|\frac{\partial}{\partial r} u(r \sigma)\right|^{2} \mathrm{~d} r\right\}\left\{\int_{\rho}^{s} r^{-n+1} \mathrm{~d} r\right\} \mathrm{d} s \mathrm{~d} S(\sigma) \\
& \leq 2 \int_{\Sigma_{x}} \int_{\rho}^{r_{x}(\sigma)}\left\{\int_{\rho}^{r_{x}(\sigma)} r^{n-1}\left|\frac{\partial}{\partial r} u(r \sigma)\right|^{2} \mathrm{~d} r\right\}\left\{s^{n-1} \int_{\rho}^{s} r^{-n+1} \mathrm{~d} r\right\} \mathrm{d} s \mathrm{~d} S(\sigma) .
\end{aligned}
$$


An easy calculation and definition (2.2) of $h_{x}$ yield

$$
\begin{aligned}
\int_{\rho}^{r_{x}(\sigma)}\left\{s \int_{\rho}^{s} r^{-1} \mathrm{~d} r\right\} \mathrm{d} s & =\int_{\rho}^{r_{x}(\sigma)}\{s \ln s-s \ln \rho\} \mathrm{d} s \\
& =\frac{1}{2} r_{x}(\sigma)^{2} \ln \left(\frac{r_{x}(\sigma)}{\rho}\right)-\frac{1}{4} r_{x}(\sigma)^{2}+\frac{1}{4} \rho^{2} \\
& \leq \frac{1}{2} h_{x}^{2} \ln \left(\frac{h_{x}}{\rho}\right)-\frac{1}{4} h_{x}^{2}+\frac{1}{4} \rho^{2}, \quad \forall \sigma \in \Sigma_{x}
\end{aligned}
$$

and, for $n \geq 3$,

$$
\begin{aligned}
\int_{\rho}^{r_{x}(\sigma)}\left\{s^{n-1} \int_{\rho}^{s} r^{-n+1} \mathrm{~d} r\right\} \mathrm{d} s & =\int_{\rho}^{r_{x}(\sigma)} \frac{s^{n-1}}{n-2}\left[\rho^{2-n}-s^{2-n}\right] \mathrm{d} s \\
& =\frac{1}{n-2}\left\{\frac{1}{n} r_{x}(\sigma)^{n} \rho^{2-n}-\frac{1}{2} r_{x}(\sigma)^{2}-\frac{1}{n} \rho^{2}+\frac{1}{2} \rho^{2}\right\} \\
& \leq \frac{1}{n-2}\left\{\frac{1}{n} h_{x}^{n} \rho^{2-n}-\frac{1}{2} h_{x}^{2}+\frac{n-2}{2 n} \rho^{2}\right\}, \quad \forall \sigma \in \Sigma_{x} .
\end{aligned}
$$

Since $\left|\frac{\partial}{\partial r} u(r \sigma)\right| \leq|\nabla u(r \sigma)|$, we therefore obtain

$$
S_{1} \leq \widetilde{K}_{n}\left(\frac{h_{x}}{\rho}\right) h_{x}^{2}\|\nabla u\|_{2 ; \omega_{x} \backslash B_{\rho}}^{2}
$$

with

$$
\widetilde{K}_{n}(z):= \begin{cases}\ln (z)-\frac{1}{2}+\frac{1}{2} z^{-2}, & \text { if } n=2, \\ \frac{2}{n-2}\left\{\frac{1}{n} z^{n-2}-\frac{1}{2}+\frac{n-2}{2 n} z^{-2}\right\}, & \text { if } n \geq 3 .\end{cases}
$$

Next, we estimate the boundary integral in (4.4):

$$
\begin{aligned}
\|u\|_{2 ; \partial B_{\rho}}^{2}= & \int_{\Sigma_{x}} \rho^{n-1}|u(\rho \sigma)|^{2} \mathrm{~d} S(\sigma) \\
= & \int_{\Sigma_{x}} \rho^{n-1}\left|\int_{0}^{\rho} \frac{\partial}{\partial r}\left[\left(\frac{r}{\rho}\right)^{n} u(r \sigma)\right] \mathrm{d} r\right|^{2} \mathrm{~d} S(\sigma) \\
= & \int_{\Sigma_{x}} \rho^{n-1}\left|\int_{0}^{\rho}\left(\frac{r}{\rho}\right)^{n} \frac{\partial}{\partial r} u(r \sigma)+n \frac{r^{n-1}}{\rho^{n}} u(r \sigma) \mathrm{d} r\right|^{2} \mathrm{~d} S(\sigma) \\
\leq & \frac{2}{\rho^{n+1}} \int_{\Sigma_{x}}\left\{\left|\int_{0}^{\rho} r^{n} \frac{\partial}{\partial r} u(r \sigma) \mathrm{d} r\right|^{2}+\left|\int_{0}^{\rho} n r^{n-1} u(r \sigma) \mathrm{d} r\right|^{2}\right\} \mathrm{d} S(\sigma) \\
\leq & \frac{2}{\rho^{n+1}} \int_{\Sigma_{x}}\left\{\int_{0}^{\rho} r^{n-1}\left|\frac{\partial}{\partial r} u(r \sigma)\right|^{2} \mathrm{~d} r \int_{0}^{\rho} r^{n+1} \mathrm{~d} r\right. \\
& \left.+n^{2} \int_{0}^{\rho} r^{n-1}|u(r \sigma)|^{2} \mathrm{~d} r \int_{0}^{\rho} r^{n-1} \mathrm{~d} r\right\} \mathrm{d} S(\sigma) \\
= & \frac{2}{\rho^{n+1}} \int_{\Sigma_{x}}\left\{\frac{\rho^{n+2}}{n+2} \int_{0}^{\rho} r^{n-1}\left|\frac{\partial}{\partial r} u(r \sigma)\right|^{2} \mathrm{~d} r+n \rho^{n} \int_{0}^{\rho} r^{n-1}|u(r \sigma)|^{2} \mathrm{~d} r\right\} \mathrm{d} S(\sigma) \\
\leq & \frac{2 \rho}{n+2}\|\nabla u\|_{2 ; B_{\rho}}^{2}+\frac{2 n}{\rho}\|u\|_{2 ; B_{\rho}}^{2} .
\end{aligned}
$$


Here, we have used the Cauchy-Schwarz inequality and the estimate $\left|\frac{\partial}{\partial r} u(r \sigma)\right| \leq|\nabla u(r \sigma)|$. Combining estimates (4.2-4.7) and recalling definition (2.7), we arrive at

$$
\begin{aligned}
\|u\|_{2 ; \omega_{x}}^{2} \leq & \|u\|_{2 ; B_{\rho}}^{2}+\widetilde{K}_{n}\left(\frac{h_{x}}{\rho}\right) h_{x}^{2}\|\nabla u\|_{2 ; \omega_{x} \backslash B_{\rho}}^{2} \\
& +\frac{2 \rho}{n}\left[\left(\frac{h_{x}}{\rho}\right)^{n}-1\right]\left\{\frac{2 \rho}{n+2}\|\nabla u\|_{2 ; B_{\rho}}^{2}+\frac{2 n}{\rho}\|u\|_{2 ; B_{\rho}}^{2}\right\} \\
= & {\left[4\left(\frac{h_{x}}{\rho}\right)^{n}-3\right]\|u\|_{2 ; B_{\rho}}^{2} } \\
& +\max \left\{\widetilde{K}_{n}\left(\frac{h_{x}}{\rho}\right), \frac{4}{n(n+2)}\left[\left(\frac{h_{x}}{\rho}\right)^{n-2}-\left(\frac{\rho}{h_{x}}\right)^{2}\right]\right\} h_{x}^{2}\|\nabla u\|_{2 ; \omega_{x}}^{2} \\
= & K_{1, n}\left(\frac{h_{x}}{\rho}\right)\left(\frac{h_{x}}{\rho}\right)^{2}\|u\|_{2 ; B_{\rho}}^{2}+K_{2, n}\left(\frac{h_{x}}{\rho}\right) h_{x}^{2}\|\nabla u\|_{2 ; \omega_{x}}^{2} .
\end{aligned}
$$

Since $0<\rho<\rho_{x}$ was arbitrary, this establishes the assertion.

Lemma 4.1 enables us to determine the quantity $\widetilde{c}_{P, x}^{(k)}$ by computing the corresponding quantities for $n$-dimensional balls and segments thereof.

Lemma 4.2. For any $x \in \mathcal{N}_{h}$ and any $r \in \mathbb{R}_{+}^{*}$ set

$$
c_{P, x}(r):=\sup \left\{\frac{\|v\|_{2 ; B_{\Sigma_{x}}(r)}}{\|\nabla v\|_{2 ; B_{\Sigma_{x}}(r)}}: v \in H^{1}\left(B_{\Sigma_{x}}(r)\right), \int_{B_{\Sigma_{x}}(r)} v=0\right\}
$$

We then have for all $r \in \mathbb{R}_{+}^{*}$

$$
c_{P, x}(r)=r c_{P, x} .
$$

Here, $c_{P, x}=c_{P, x}$ (1) is given by equation (2.5). Moreover estimate (2.6) holds for $c_{P, x}$.

Proof. Let $u \in H^{1}\left(B_{\Sigma_{x}}\right)$ with $\int_{B_{\Sigma_{x}}} u=0$ and $r \in \mathbb{R}_{+}^{*}$. Set

$$
v(y):=u\left(r^{-1} y\right), \quad \forall y \in B_{\Sigma_{x}}(r) .
$$

An easy calculation yields

$$
\begin{aligned}
\int_{B_{\Sigma_{x}}(r)} v(y) & =r^{n} \int_{B_{\Sigma_{x}}} u=0, \\
\|v\|_{2 ; B_{\Sigma_{x}}(r)} & =r^{\frac{n}{2}}\|u\|_{2 ; B_{\Sigma_{x}}}, \\
\|\nabla v\|_{2 ; B_{\Sigma_{x}}(r)} & =r^{\frac{n}{2}-1}\|\nabla u\|_{2 ; B_{\Sigma_{x}}} .
\end{aligned}
$$

Hence, we have

$$
\frac{\|v\|_{2 ; B_{\Sigma_{x}}(r)}}{\|\nabla v\|_{2 ; B_{\Sigma_{x}}(r)}}=r \frac{\|u\|_{2 ; B_{\Sigma_{x}}}}{\|\nabla u\|_{2 ; B_{\Sigma_{x}}}}
$$

This proves that $r c_{P, x} \leq c_{P, x}(r)$. Interchanging the roles of $u$ and $v$ proves the opposite inequality and establishes equation (4.8). 
In order to prove estimate (2.6) consider first an interior vertex $x$. Since in this case $B_{\Sigma_{x}}$ is the unit ball, we know from [9] that

$$
c_{P, x}^{-1}=\inf \left\{x \in \mathbb{R}_{+}^{*}: x J_{\frac{n}{2}}^{\prime}(x)-\frac{n-2}{2} J_{\frac{n}{2}}(x)=0\right\},
$$

where $J_{n / 2}$ is the Bessel function of order $n / 2$. From [1] we conclude that the first extremum of $J_{1}$ lies in the open interval $(1.8,1.9)$. This proves the first estimate of $(2.6)$. The second one follows from [9].

Next, consider a boundary vertex $x$. If $B_{\Sigma_{x}}$ is convex, i.e. $x \in \mathcal{N}_{h, N}^{(c)} \cup \mathcal{N}_{h, D}^{(c)}$, the corresponding estimate of (2.6) follows from [9]. Thus we remain with the non-convex case, i.e. $x \in \mathcal{N}_{h, N}^{(n c)} \cup \mathcal{N}_{h, D}^{(n c)}$. Since $\Omega$ has a Lipschitz boundary, $B_{\Sigma_{x}}$ satisfies an exterior cone-condition. Hence, there is an $\alpha \in(0, \pi)$ and a cone $C$ with base 0 and opening angle $\alpha$ such that $C \subset \mathbb{R}^{n} \backslash B_{\Sigma_{x}}$. Without loss of generality we may assume that the positive $x_{1}$-axis is the axis of symmetry of $C$. Then one easily checks that $B_{\Sigma_{x}}$ is star-shaped w.r.t. $-\frac{1}{2} e_{1}$ and that

$$
\begin{aligned}
h & :=\max _{y \in \partial B_{\Sigma_{x}}}\left|y+\frac{1}{2} e_{1}\right|_{2}=\frac{1}{2}\left\{5+4 \cos \frac{\alpha}{2}\right\}^{1 / 2} \leq \frac{3}{2}, \\
\rho & :=\min _{y \in \partial B_{\Sigma_{x}}}\left|y+\frac{1}{2} e_{1}\right|_{2}=\frac{1}{2},
\end{aligned}
$$

where $|\cdot|_{2}$ is the Euclidean norm in $\mathbb{R}^{n}$. Consider an arbitrary function $u \in H^{1}\left(B_{\Sigma_{x}}\right)$ with $\int_{B_{\Sigma_{x}}} u=0$. Denote by $B$ the ball with centre $-\frac{1}{2} e_{1}$ and radius $\rho$ and set $\bar{u}:=u-\frac{1}{\mu_{n}(B)} \int_{B} u$. Now we may apply relation (4.8) and Lemma 4.1 with $\kappa_{1, x}, h_{x}, \rho_{x}, \omega_{x}$, and $B_{\Sigma_{x}}$ replaced by $\kappa:=\frac{h}{\rho}, h, \rho, B_{\Sigma_{x}}$, and $B$, resp. and obtain

$$
\begin{aligned}
\|u\|_{2 ; B_{\Sigma_{x}}} & =\inf _{p \in \mathbb{P}_{0}}\|u-p\|_{2 ; B_{\Sigma_{x}}} \leq\|\bar{u}\|_{2 ; B_{\Sigma_{x}}} \\
& \leq\left\{\widehat{c}_{P}^{2} K_{1, n}(\kappa)+K_{2, n}(\kappa)\right\}^{1 / 2} h\|\nabla u\|_{2 ; B_{\Sigma_{x}}} .
\end{aligned}
$$

Here $\widehat{c}_{P}$ denotes the Poincaré constant (2.5) for the unit ball. It is bounded by the first two estimates of (2.6). Since $u$ was arbitrary this proves that

$$
c_{P, x} \leq\left\{\widehat{c}_{P}^{2} K_{1, n}(\kappa)+K_{2, n}(\kappa)\right\}^{1 / 2} h .
$$

This establishes the last three estimates of (2.6). For completeness we note that one can also use the results of [4] to estimate $c_{P, x}$ for re-entrant corners, i.e. $x \in \mathcal{N}_{h, D}^{(n c)} \cup \mathcal{N}_{h, N}^{(n c)}$. The resulting upper bounds, however, tend to infinity when the exterior angle $\alpha$ approaches zero.

Lemmas 4.1 and 4.2 enable us to determine the quantity $\widetilde{c}_{P, x}^{(k)}$ of $(4.1)$.

Lemma 4.3. For all $x \in \mathcal{N}_{h}$ and all $u \in H^{1}\left(\omega_{x}\right)$ we have

$$
\left\|u-\pi_{x}^{(0)} u\right\|_{2 ; \omega_{x}} \leq c_{x} h_{x}\|\nabla u\|_{2 ; \omega_{x}}
$$

where $c_{x}$ is given by equation (2.7)

Proof. As in the proof of Lemma 4.1 we may assume that the vertex $x$ is the origin. If $\omega_{x}$ is convex, the result follows from [9]. For the general case set

$$
\bar{u}:=u-\frac{1}{\mu_{n}\left(B_{\Sigma_{x}}\left(\rho_{x}\right)\right)} \int_{B_{\Sigma_{x}}\left(\rho_{x}\right)} u .
$$


From Lemma 4.1 we obtain

$$
\begin{aligned}
\left\|u-\pi_{x}^{(0)} u\right\|_{2 ; \omega_{x}}^{2} & \leq\|\bar{u}\|_{2 ; \omega_{x}}^{2} \\
& \leq K_{1, n}\left(\kappa_{1, x}\right) \kappa_{1, x}^{2}\|\bar{u}\|_{2 ; B_{\Sigma_{x}}\left(\rho_{x}\right)}^{2}+K_{2, n}\left(\kappa_{1, x}\right) h_{x}^{2}\|\nabla \bar{u}\|_{2 ; \omega_{x}}^{2} .
\end{aligned}
$$

Since $\int_{B_{\Sigma_{x}}\left(\rho_{x}\right)} \bar{u}=0$, Lemma 4.2 , on the other hand, yields

$$
\|\bar{u}\|_{2 ; B_{\Sigma_{x}}\left(\rho_{x}\right)} \leq c_{P, x} \rho_{x}\|\nabla \bar{u}\|_{2 ; B_{\Sigma_{x}}\left(\rho_{x}\right)} .
$$

Since $\nabla \bar{u}=\nabla u$, this proves the assertion.

Lemma 4.4. For all $x \in \mathcal{N}_{h}$ and all $u \in H^{2}\left(\omega_{x}\right)$ we have

$$
\left\|u-\pi_{x}^{(1)} u\right\|_{2 ; \omega_{x}} \leq c_{x}^{2} h_{x}^{2}\left\|\nabla^{2} u\right\|_{2 ; \omega_{x}}
$$

and

$$
\left\|\nabla\left(u-\pi_{x}^{(1)} u\right)\right\|_{2, \omega_{x}} \leq c_{x} h_{x}\left\|\nabla^{2} u\right\|_{2 ; \omega_{x}}
$$

where $c_{x}$ is given by equation (2.7).

Proof. Since $\partial_{i}\left(\pi_{x}^{(1)} u\right)=\pi_{x}^{(0)}\left(\partial_{i} u\right)$ holds for all $1 \leq i \leq n$, the second estimate follows from Lemma 4.3. Set $v:=u-\sum_{1 \leq i \leq n} a_{i} x_{i}$. Then we have $u-\pi_{x}^{(1)} u=v-\pi_{x}^{(0)} v$ and $\nabla\left(u-\pi_{x}^{(1)} u\right)=\nabla v$. Hence, the first estimate follows from the second one and from Lemma 4.3.

Lemmas 4.3 and 4.4 in particular imply that

$$
\begin{aligned}
\inf _{p \in \mathbb{P}_{m}}\left\|\nabla^{j}(u-p)\right\|_{2 ; \omega_{x}} \leq & c_{x}^{m-j+1} h_{x}^{m-j+1}\left\|\nabla^{m+1} u\right\|_{2 ; \omega_{x}} \\
& \forall u \in H^{m+1}\left(\omega_{x}\right), m \in\{0,1\}, j \in\{0, m\} .
\end{aligned}
$$

This estimate should be compared with the result of [8] which reads

$$
\begin{gathered}
\inf _{p \in \mathbb{P}_{m}}\left\|\nabla^{j}(u-p)\right\|_{2 ; \omega_{x}} \leq C_{m, j, n}\left(\frac{h_{x}}{\rho}\right)^{\frac{n}{2}}\left(2 h_{x}\right)^{m-j+1}\left\|\nabla^{m+1} u\right\|_{2 ; \omega_{x}} \\
\forall u \in H^{m+1}\left(\omega_{x}\right), m \in \mathbb{N}, 0 \leq j \leq m
\end{gathered}
$$

with

$$
C_{m, j, n}=\left\{\begin{array}{lll}
2 n^{1 / 2} & \text { if } m=0, & j=0 \\
\sqrt{8} n & \text { if } m=1, & j=0 \\
2 n^{3 / 2} & \text { if } m=1, & j=1
\end{array}\right.
$$

Here, the parameter $\rho$ can be chosen in a maximal way such that $\omega_{x}$ contains a ball of radius $\rho$ and is star-shaped w.r.t. every point in that ball. If $x$ is an interior vertex, we may choose $\rho=\rho_{x}$ and obtain $h_{x} / \rho=\kappa_{1, x}$. If $n=2$ and $x$ is a boundary vertex, an easy geometrical argument shows that

$$
\rho=\frac{\sin (\alpha / 2)}{1+\sin (\alpha / 2)} \rho_{x} \quad \text { and } \quad \frac{h_{x}}{\rho}=\frac{1+\sin (\alpha / 2)}{\sin (\alpha / 2)} \kappa_{1, x} .
$$

Here, $\alpha$ is the interior angle of $\Omega$ at the vertex $x$. Note, that the resulting bound tends to infinity if $\alpha$ approaches 0 or $2 \pi$. 


\section{Proof of Proposition 2.1}

In order to reduce technical difficulties with the treatment of Dirichlet boundary conditions, we introduce a modification $\bar{P}_{h}$ of $P_{h}$ which ignores boundary conditions and which is given by

$$
\bar{P}_{h} u=\sum_{x \in \mathcal{N}_{h}} \lambda_{x} \pi_{x}^{(0)} u
$$

Fix an arbitrary simplex $T \in \mathcal{T}_{h}$ and an arbitrary face $E \in \mathcal{E}(T)$. Since $\sum_{x \in \mathcal{N}(T)} \lambda_{x}=1$ on $T$, we have

$$
\begin{aligned}
\left\|u-\bar{P}_{h} u\right\|_{2 ; T} & =\left\|\sum_{x \in \mathcal{N}(T)} \lambda_{x}\left(u-\pi_{x}^{(0)} u\right)\right\|_{2 ; T} \\
& \leq \sum_{x \in \mathcal{N}(T)}\left\|\lambda_{x}\right\|_{\infty ; T}\left\|u-\pi_{x}^{(0)} u\right\|_{2 ; T} \\
& =\sum_{x \in \mathcal{N}(T)}\left\|u-\pi_{x}^{(0)} u\right\|_{2 ; T} .
\end{aligned}
$$

From Lemma 4.3 on the other hand, we obtain for all $x \in \mathcal{N}(T)$

$$
\left\|u-\pi_{x}^{(0)} u\right\|_{2 ; T} \leq\left\|u-\pi_{x}^{(0)} u\right\|_{2 ; \omega_{x}} \leq c_{x} h_{x}\|\nabla u\|_{2 ; \omega_{x}} .
$$

If $\mathcal{N}(T) \cap \mathcal{N}_{h, D}=\emptyset$, the functions $\bar{P}_{h} u$ and $P_{h} u$ coincide on $T$. Hence, equations $(5.1,5.2)$ prove that $c_{T ; x}^{(P)}=c_{x}$ if $x \in \mathcal{N}_{h, \Omega} \cup \mathcal{N}_{h, N}$.

Next, we consider the case that $\mathcal{N}(T) \cap \mathcal{N}_{h, D} \neq \emptyset$. We then have

$$
\begin{aligned}
\left\|P_{h} u-\bar{P}_{h} u\right\|_{2 ; T} & =\left\|\sum_{x \in \mathcal{N}(T) \cap \mathcal{N}_{h, D}} \lambda_{x} \pi_{x}^{(0)} u\right\|_{2 ; T} \\
& \leq \sum_{x \in \mathcal{N}(T) \cap \mathcal{N}_{h, D}}\left\|\lambda_{x}\right\|_{2 ; T}\left|\pi_{x}^{(0)} u\right| .
\end{aligned}
$$

Consider an $x \in \mathcal{N}(T) \cap \mathcal{N}_{h, D}$. Choose a face $E_{x} \in \mathcal{E}_{h, D}$ such that $x \in \mathcal{N}\left(E_{x}\right)$ and a simplex $T_{x} \in \mathcal{T}_{h}$ such that $E_{x} \in \mathcal{E}\left(T_{x}\right)$ and $x \in \mathcal{N}\left(T_{x}\right)$. Since $u$ vanishes on $E_{x}$ we have

$$
\begin{aligned}
\left\|\lambda_{x}\right\|_{2 ; T}\left|\pi_{x}^{(0)} u\right| & =\frac{\left\|\lambda_{x}\right\|_{2 ; T}}{\left\|\lambda_{x}\right\|_{2 ; E_{x}}}\left\|\lambda_{x} \pi_{x}^{(0)} u\right\|_{2 ; E_{x}} \\
& =\frac{\left\|\lambda_{x}\right\|_{2 ; T}}{\left\|\lambda_{x}\right\|_{2 ; E_{x}}}\left\|\lambda_{x}\left(\pi_{x}^{(0)} u-u\right)\right\|_{2 ; E_{x}}
\end{aligned}
$$

Since

$$
\left\|\lambda_{x}\right\|_{2 ; T}=\left\{\frac{2 n !}{(n+2) !} \mu_{n}(T)\right\}^{1 / 2}, \quad\left\|\lambda_{x}\right\|_{2 ; E_{x}}=\left\{\frac{2(n-1) !}{(n+1) !} \mu_{n-1}\left(E_{x}\right)\right\}^{1 / 2}
$$

Lemma 3.2 applied to $u-\pi_{x}^{(0)} u$ yields

$$
\begin{aligned}
\left\|\lambda_{x}\right\|_{2 ; T}\left|\pi_{x}^{(0)} u\right| & \leq\left\{\frac{2 n !}{(n+2) !} \mu_{n}(T) \frac{(n+1) !}{2(n-1) !} \frac{1}{\mu_{n_{x}}\left(E_{x}\right)} \frac{1}{n} \frac{\mu_{n-1}\left(E_{x}\right)}{\mu_{n}\left(T_{x}\right)}\right\}^{1 / 2}\left\{\left\|u-\pi_{x}^{(0)} u\right\|_{2 ; T_{x}}+h_{x}\|\nabla u\|_{2 ; T_{x}}\right\} \\
& =\left\{\frac{1}{n+2} \frac{\mu_{n}(T)}{\mu_{n}\left(T_{x}\right)}\right\}^{1 / 2}\left\{\left\|u-\pi_{x}^{(0)} u\right\|_{2 ; T_{x}}+h_{x}\|\nabla u\|_{2 ; T_{x}}\right\} .
\end{aligned}
$$


Combining estimates $(5.3,5.6)$ with inequalities $(5.1,5.2)$ we finally arrive at

$$
\begin{aligned}
\left\|u-P_{h} u\right\|_{2 ; T} & \leq\left\|u-\bar{P}_{h} u\right\|_{2 ; T}+\left\|\bar{P}_{h} u-P_{h} u\right\|_{2 ; T} \\
& \leq \sum_{x \in \mathcal{N}(T)} c_{x} h_{x}\|\nabla u\|_{2 ; \omega_{x}}+\sum_{x \in \mathcal{N}(T) \cap \mathcal{N}_{h, D}}\left\{\frac{1}{n+2} \frac{\mu_{n}(T)}{\mu_{n}\left(T_{x}\right)}\right\}^{1 / 2}\left\{c_{x}+1\right\} h_{x}\|\nabla u\|_{2 ; \omega_{x}} .
\end{aligned}
$$

Recalling definition (2.4), this proves the estimate for $c_{T ; x}^{(P)}$ in the case $\mathcal{N}(T) \cap \mathcal{N}_{h, D} \neq \emptyset$.

Next we consider the face $E$. Similarly to estimate (5.1) we have

$$
\left\|u-\bar{P}_{h} u\right\|_{2 ; E} \leq \sum_{x \in \mathcal{N}(E)}\left\|\lambda_{x}\left(u-\pi_{x}^{(0)} u\right)\right\|_{2 ; E}
$$

Lemma 3.2 applied to $u-\pi_{x}^{(0)} u$ yields for all $x \in \mathcal{N}(E)$

$$
\left\|\lambda_{x}\left(u-\pi_{x}^{(0)} u\right)\right\|_{2 ; E} \leq\left\{\frac{1}{n} \frac{\mu_{n-1}(E)}{\mu_{n}(T)}\right\}^{1 / 2}\left\{\left\|u-\pi_{x}^{(0)} u\right\|_{2 ; T}+h_{x}\|\nabla u\|_{2 ; T}\right\}
$$

Since $\bar{P}_{h} u$ and $P_{h} u$ coincide on $E$ if $\mathcal{N}(E) \cap \mathcal{N}_{h, D}=\emptyset$, estimates $(5.2,5.7,5.8)$ prove the estimate for $c_{E ; x}^{(P)}$ in this case.

Finally, assume that $\mathcal{N}(E) \cap \mathcal{N}_{h, D} \neq \emptyset$. As in estimates (5.3-5.6) we obtain with the same notations

$$
\left\|P_{h} u-\bar{P}_{h} u\right\|_{2 ; E} \leq \sum_{x \in \mathcal{N}(E) \cap \mathcal{N}_{h, D}}\left\|\lambda_{x}\right\|_{2 ; E}\left|\pi_{x}^{(0)} u\right|
$$

and

$$
\begin{aligned}
\left\|\lambda_{x}\right\|_{2 ; E}\left|\pi_{x}^{(0)} u\right| & =\frac{\left\|\lambda_{x}\right\|_{2 ; E}}{\left\|\lambda_{x}\right\|_{2 ; E_{x}}}\left\|\lambda_{x} \pi_{x}^{(0)} u\right\|_{2 ; E_{x}} \\
& =\frac{\left\|\lambda_{x}\right\|_{2 ; E}}{\left\|\lambda_{x}\right\|_{2 ; E_{x}}}\left\|\lambda_{x}\left(\pi_{x}^{(0)} u-u\right)\right\|_{2 ; E_{x}} \\
& =\left\{\frac{\mu_{n-1}(E)}{\mu_{n-1}\left(E_{x}\right)}\right\}^{1 / 2}\left\|\lambda_{x}\left(\pi_{x}^{(0)} u-u\right)\right\|_{2 ; E_{x}} \\
& \leq\left\{\frac{1}{n} \frac{\mu_{n-1}(E)}{\mu_{n}\left(T_{x}\right)}\right\}^{1 / 2}\left\{\left\|u-\pi_{x}^{(0)} u\right\|_{2 ; T_{x}}+h_{x}\|\nabla u\|_{2 ; T_{x}}\right\} .
\end{aligned}
$$

Combining estimates $(5.2,5.8-5.10)$ we arrive at

$$
\begin{aligned}
\left\|u-P_{h} u\right\|_{2 ; E} \leq & \sum_{x \in \mathcal{N}(E)}\left\{\frac{1}{n} \frac{\mu_{n-1}(E)}{\mu_{n}(T)}\right\}^{1 / 2}\left\{c_{x}+1\right\} h_{x}\|\nabla u\|_{2 ; \omega_{x}} \\
& +\sum_{x \in \mathcal{N}(E) \cap \mathcal{N}_{h, D}}\left\{\frac{1}{n} \frac{\mu_{n-1}(E)}{\mu_{n}\left(T_{x}\right)}\right\}^{1 / 2}\left\{c_{x}+1\right\} h_{x}\|\nabla u\|_{2 ; \omega_{x}} .
\end{aligned}
$$

Recalling definition (2.4), this completes the proof of Proposition 2.1. 


\section{Proof of Proposition 2.2}

The proof of Proposition 2.2 is very similar to the one of Proposition 2.1. Again, we introduce a modification $\bar{Q}_{h}$ of $Q_{h}$ which ignores boundary conditions and which is given by

$$
\bar{Q}_{h} u=\sum_{x \in \mathcal{N}_{h}} \lambda_{x} \pi_{x}^{(1)} u(x)
$$

Fix an arbitrary $T \in \mathcal{T}_{h}$ and an arbitrary $E \in \mathcal{E}(T)$. Let $x_{1}$ be any vertex of $T$. We then have

$$
\left\|u-\bar{Q}_{h} u\right\|_{2 ; T} \leq\left\|u-\pi_{x_{1}}^{(1)} u\right\|_{2 ; T}+\left\|\pi_{x_{1}}^{(1)} u-\bar{Q}_{h} u\right\|_{2 ; T}
$$

and

$$
\begin{aligned}
\left\|\pi_{x_{1}}^{(1)} u-\bar{Q}_{h} u\right\|_{2 ; T} & =\left\|\sum_{x \in \mathcal{N}(T)} \lambda_{x}\left[\pi_{x_{1}}^{(1)} u(x)-\pi_{x}^{(1)} u(x)\right]\right\|_{2 ; T} \\
& \leq \sum_{x \in \mathcal{N}(T) \backslash\left\{x_{1}\right\}}\left\|\lambda_{x}\right\|_{2 ; T}\left\|\pi_{x_{1}}^{(1)} u-\pi_{x}^{(1)} u\right\|_{\infty ; T} .
\end{aligned}
$$

Let $\widehat{p} \in \mathbb{P}_{1}$ be arbitrary and set

$$
z:=\left(\widehat{p}\left(e_{1}\right), \ldots, \widehat{p}\left(e_{n+1}\right)\right) \in \mathbb{R}^{n+1}
$$

We then have

$$
\|\widehat{p}\|_{\infty ; \widehat{T}}=|z|_{\infty}
$$

and

$$
\|\widehat{p}\|_{2 ; \widehat{T}}=\left\{z^{t} A z\right\}^{1 / 2} \geq \lambda_{\min }(A)^{1 / 2}|z|_{2} \geq \lambda_{\min }(A)^{1 / 2}|z|_{\infty} .
$$

Here, $\lambda_{\min }(A)$ denotes the minimal eigenvalue of $A$ and the $(n+1) \times(n+1)$ matrix $A$ has diagonal entries $2 /(n+2)$ ! and non-diagonal entries $1 /(n+2)$ !. Hence, $1 /(n+2)$ ! is an $n$-fold eigenvalue of $A$ and $n+2 /(n+2)$ ! is a simple eigenvalue of $A$. Thus we have

$$
\|\widehat{p}\|_{\infty ; \widehat{T}} \leq \sqrt{(n+2) !}\|\widehat{p}\|_{2 ; \widehat{T}} .
$$

Let $p \in \mathbb{P}_{1}$ be arbitrary and consider an affine transformation $F$ of $\widehat{T}$ onto $T$. With $\widehat{p}:=p \circ F$ we then have

$$
\|p\|_{\infty ; T}=\|\widehat{p}\|_{\infty ; \widehat{T}} \leq \sqrt{(n+2) !}\|\widehat{p}\|_{2 ; \widehat{T}}=\left\{(n+2) ! \frac{\mu_{n}(\widehat{T})}{\mu_{n}(T)}\right\}^{1 / 2}\|p\|_{2 ; T}
$$

Combining estimates $(5.5,6.3)$ we conclude that

$$
\begin{aligned}
\left\|\lambda_{x}\right\|_{2 ; T}\left\|\pi_{x_{1}}^{(1)} u-\pi_{x}^{(1)} u\right\|_{\infty ; T} & \leq\left\{2 n ! \mu_{n}(\widehat{T})\right\}^{1 / 2}\left\|\pi_{x_{1}}^{(1)} u-\pi_{x}^{(1)} u\right\|_{2 ; T} \\
& \leq \sqrt{2}\left\{\left\|u-\pi_{x_{1}}^{(1)} u\right\|_{2 ; T}+\left\|u-\pi_{x}^{(1)} u\right\|_{2 ; T}\right\}
\end{aligned}
$$

Since $x_{1}$ was an arbitrary vertex of $T$, inequalities $(6.1,6.2,6.4)$ imply that

$$
\left\|u-\bar{Q}_{h} u\right\|_{2 ; T} \leq\left\{\sqrt{2}+\frac{1}{n+1}\right\} \sum_{x \in \mathcal{N}(T)}\left\|u-\pi_{x}^{(1)} u\right\|_{2 ; T} .
$$


From Lemma 4.4 on the other hand, we get for all $x \in \mathcal{N}(T)$

$$
\left\|u-\pi_{x}^{(1)} u\right\|_{2 ; T} \leq\left\|u-\pi_{x}^{(1)} u\right\|_{2 ; \omega_{x}} \leq c_{x}^{2} h_{x}^{2}\left\|\nabla^{2} u\right\|_{2 ; \omega_{x}} .
$$

This proves the estimate for $c_{T ; x}^{(Q)}$ in the case $\mathcal{N}(T) \cap \mathcal{N}_{h, D}=\emptyset$. Now, assume that $\mathcal{N}(T) \cap \mathcal{N}_{h, D} \neq \emptyset$. We then have

$$
\begin{aligned}
\left\|Q_{h} u-\bar{Q}_{h} u\right\|_{2 ; T} & =\left\|\sum_{x \in \mathcal{N}(T) \cap \mathcal{N}_{h, D}} \lambda_{x} \pi_{x}^{(1)} u(x)\right\|_{2 ; T} \\
& \leq \sum_{x \in \mathcal{N}(T) \cap \mathcal{N}_{h, D}}\left\|\lambda_{x}\right\|_{2 ; T}\left|\pi_{x}^{(1)} u(x)\right|
\end{aligned}
$$

Retaining the notations of Section 5 and using estimate (6.3) with $n, T$, and $\widehat{T}$ replaced by $n-1, E_{x}$, and $\widehat{E}_{1}$, respectively, we conclude that for all $x \in \mathcal{N}(T) \cap \mathcal{N}_{h, D}$

$$
\begin{aligned}
\left|\pi_{x}^{(1)} u(x)\right| & \leq\left\|\pi_{x}^{(1)} u\right\|_{\infty ; E_{x}} \\
& \leq\left\{(n+1) ! \frac{\mu_{n-1}\left(\widehat{E}_{1}\right)}{\mu_{n-1}\left(E_{x}\right)}\right\}^{1 / 2}\left\|\pi_{x}^{(1)} u\right\|_{2 ; E_{x}} \\
& =\left\{(n+1) ! \frac{\mu_{n-1}\left(\widehat{E}_{1}\right)}{\mu_{n-1}\left(E_{x}\right)}\right\}^{1 / 2}\left\|u-\pi_{x}^{(1)} u\right\|_{2 ; E_{x}} .
\end{aligned}
$$

Here, we have used that $u$ vanishes on $E_{x}$. Let $T_{x} \in \mathcal{T}_{h}$ be such that $E_{x} \in \mathcal{E}\left(T_{x}\right)$ and $x \in \mathcal{N}\left(T_{x}\right)$. Applying Lemma 3.2 to $u-\pi_{x}^{(1)} u$ and using Lemma 4.4 we get

$$
\begin{aligned}
\left\|u-\pi_{x}^{(1)} u\right\|_{2 ; E_{x}} & \leq\left\{n \frac{\mu_{n-1}\left(E_{x}\right)}{\mu_{n}\left(T_{x}\right)}\right\}^{1 / 2}\left\{\left\|u-\pi_{x}^{(1)} u\right\|_{2 ; T_{x}}+h_{T_{x}}\left\|\nabla\left(u-\pi_{x}^{(1)} u\right)\right\|_{2 ; T_{x}}\right\} \\
& \leq\left\{n \frac{\mu_{n-1}\left(E_{x}\right)}{\mu_{n}\left(T_{x}\right)}\right\}^{1 / 2}\left\{c_{x}^{2}+\kappa_{2, x} c_{x}\right\} h_{x}^{2}\left\|\nabla^{2} u\right\|_{2 ; \omega_{x}} .
\end{aligned}
$$

Combining estimates $(6.5-6.9,5.5)$, establishes the estimate for $c_{T ; x}^{(Q)}$ in the case $\mathcal{N}(T) \cap \mathcal{N}_{h, D} \neq \emptyset$, too.

Next, we consider the face $E$. Choose an arbitrary vertex $x_{1}$ of $E$. Similarly, to estimates (6.1-6.4) we have

$$
\left\|u-\bar{Q}_{h} u\right\|_{2 ; E} \leq\left\|u-\pi_{x_{1}}^{(1)} u\right\|_{2 ; E}+\sum_{x \in \mathcal{N}(E) \backslash\left\{x_{1}\right\}}\left\|\lambda_{x}\right\|_{2 ; E}\left\|\pi_{x_{1}}^{(1)} u-\pi_{x}^{(1)} u\right\|_{\infty ; E}
$$

and, for all $x \in \mathcal{N}(E) \backslash\left\{x_{1}\right\}$,

$$
\begin{aligned}
\left\|\lambda_{x}\right\|_{2 ; E}\left\|\pi_{x_{1}}^{(1)} u-\pi_{x}^{(1)} u\right\|_{\infty ; E} & \leq\left\{\mu_{n-1}(E) \frac{2(n-1) !}{(n+1) !}(n+1) ! \frac{\mu_{n-1}\left(\widehat{E}_{1}\right)}{\mu_{n-1}(E)}\right\}^{1 / 2}\left\|\pi_{x_{1}}^{(1)} u-\pi_{x}^{(1)} u\right\|_{2 ; E} \\
& \leq \sqrt{2}\left\{\left\|u-\pi_{x_{1}}^{(1)} u\right\|_{2 ; E}+\left\|u-\pi_{x_{1}}^{(1)} u\right\|_{2 ; E}\right\}
\end{aligned}
$$

Since $x_{1} \in \mathcal{N}(E)$ was arbitrary, we obtain the following analogue of (6.5)

$$
\left\|u-\bar{Q}_{h} u\right\|_{2 ; E} \leq\left\{\sqrt{2}+\frac{1}{n}\right\} \sum_{x \in \mathcal{N}(E)}\left\|u-\pi_{x}^{(1)} u\right\|_{2 ; E}
$$


Recalling definition (2.4) of $\kappa_{4, x}$, estimates (6.9) and (6.12) establish the estimate for $c_{E ; x}^{(Q)}$ in the case $\mathcal{N}(E) \cap$ $\mathcal{N}_{h, D}=\emptyset$.

Finally, we consider the case that $\mathcal{N}(E) \cap \mathcal{N}_{h, D} \neq \emptyset$. We then have

$$
\left\|Q_{h} u-\bar{Q}_{h} u\right\|_{2 ; E} \leq \sum_{x \in \mathcal{N}(E) \cap \mathcal{N}_{h, D}}\left\|\lambda_{x}\right\|_{2 ; E}\left|\pi_{x}^{(1)} u(x)\right| .
$$

The term $\left|\pi_{x}^{(1)} u(x)\right|$ is bounded in estimates (6.8) and (6.9). The term $\left\|\lambda_{x}\right\|_{2 ; E}$ is given in equality (5.5). Combining these estimates with those for $\left\|u-\bar{Q}_{h} u\right\|_{2 ; E}$ completes the proof of Proposition 2.2.

\section{Proof of Proposition 2.4}

From Exercise 3.1.2 in [6] we know that for $m \in\{0,1\}$

$$
\left\|\nabla^{m}\left(u-\Pi_{h} u\right)\right\|_{2 ; T} \leq\left\{\sum_{x \in \mathcal{N}(T)}\left\|\nabla^{m} \lambda_{x}\right\|_{\infty ; T}\right\} \frac{2}{4-n} h_{T}^{2}\left\|\nabla^{2} u\right\|_{2 ; T}
$$

Since $T$ has $n+1$ vertices and since for all $x \in \mathcal{N}(T)$

$$
\left\|\lambda_{x}\right\|_{\infty ; T}=1,\left\|\nabla \lambda_{x}\right\|_{\infty ; T} \leq \max _{y \in \mathcal{N}(T) \backslash\{x\}} \frac{1}{|x-y|_{2}},
$$

this estimate and Lemma 3.2 prove Proposition 2.4.

\section{REFERENCES}

[1] Handbook of Mathematical Functions, M. Abramowitz and I.A. Stegun Eds., Dover Publ., New York (1965).

[2] R.A. Adams, Sobolev Spaces. Academic Press, New York (1975).

[3] L. Angermann, A posteriori Fehlerabschätzungen für Lösungen gestörter Operatorgleichungen. Habilitationsschrift, Universität Erlangen-Nürnberg (1994).

[4] J.H. Bramble and L.E. Payne, Bounds in the Neumann problem for second order uniformly elliptic operators. Pacific J. Math. $12(1962)$ 823-833.

[5] C. Carstensen and St. A. Funken, Constants in Clément-interpolation error and residual based a posteriori error estimates in finite element methods. Report 97-11, Universität Kiel (1997).

[6] Ph.G. Ciarlet, The Finite Element Method for Elliptic Problems. North Holland (1978).

[7] P. Clément, Approximation by finite element functions using local regularization. RAIRO Anal. Numér. 9 (1975) 77-84.

[8] R.G. Durán, On polynomial approximation in Sobolev spaces. SIAM J. Numer. Anal. 20 (1983) 985-988.

[9] L.E. Payne and H.F. Weinberger, An optimal Poincaré-inequality for convex domains. Arch. Rational Mech. Anal. 5 (1960) $286-292$.

[10] L.R. Scott and S. Zhang, Finite element interpolation of nonsmooth functions satisfying boundary conditions. Math. Comp. 54 (1990) 483-493.

[11] R. Verfürth, A Review of a posteriori Error Estimation and adaptive Mesh-Refinement Techniques. Teubner-Wiley, Stuttgart (1996). 